Proyecciones

Vol. 18, $\mathrm{N}^{\circ}$ 1, pp. 23-48, July 1999

Universidad Católica del Norte

Antofagasta - Chile

\title{
DIHEDRAL GROUPS ARE OF SCHOTTKY TYPE *
}

\author{
RUBÉN A. HIDALGO \\ Universidad Técnica Federico Santa María, Valparaíso-Chile
}

\begin{abstract}
We show that a dihedral group $H$ of conformal automorphisms of a closed Riemann surface $S$ can be lifted for a suitable Schotthy uniformization of $S$. In particular, this implies the existence of a suitable symplectic homology basis of $S$ for which the symplectic representation of $H$ has a simple form.
\end{abstract}

1991 Mathematics Subject Classification. Primary $30 F 10$.

*Partially supported by Projects Fondecyt 8970007, LTFSM 971223 and Presidential Science Chair on Geometry. 


\section{Introduction}

Given a closed Riemann surface $S$, retrosection theorem asserts that there are Schottky uniformizations $(\Omega, G, P: \Omega \rightarrow S)$ of $S$, that is, $G$ is a Schottky group with region of discontinuity $\Omega$ and $P: \Omega \rightarrow S$ is a Galois covering with $G$ as covering group.

The importance of Schottky uniformizations respect to the non-universal uniformizations of Riemann surfaces is given by the easy understanding of their geometry. Moreover, there is a conjecture that we can choose our Schottky groups to be classical ones, that is, there is a fundamental domain for the group $G$ consisting on $2 g$ round circles ( $g=$ the genus of the surface $S$ ). Positive evidence of this conjecture is given by some recent work by Maskit.

Classical Schottky groups permits easy computations of some complex analytic functions and/or forms.

Assume that $H$ is a group of conformal automorphisms of the surface $S$. We may ask if there is a Schottky uniformization of $S$ for which the group $H$ lifts and, if this is the case, then we say that $H$ is of Schottky type.

In [4] we have given a necessary condition, called condition (A), for $H$ to be of Schottky type. This condition turns out to be sufficient if $H$ is an abelian group (see [4] and [5]). If $H$ is isomorphic to a dihedral group, then condition (A) hold trivially.

In [6] we have shown that if $H$ acts free fixed point and it is isomorphic to a dihedral group, then it is of Schottky type. A list of Schottky uniformizations that reflect a dihedral action is also constructed in the above paper. Is natural to ask if all dihedral actions, as conformal groups, are of Schottky type. In the same paper, we constructed an example of a dihedral action of order 6 , acting on a Riemamn surface of genus 4 , and mistakable asserted not to be of Schottky type. In trying to fill out the gap in the arguments, we found that it was in fact of Schottky type. In particular, the question if every dihedral action is of Schottky type still need an answer. The main purpose of this note is to give a definitive answer to the above question.

Theorem 1. Let $S$ be a closed Riemann surface and $H$ a group of conformal automorphisms of $S$. If $H$ is isomorphic to a dihedral group, then $H$ is of Schottky type. 
In other words, theorem 1 asserts that any dihedral group of conformal automorphisms of a closed Riemann surface can be realized as a finite normal extension of a Schottky group that uniformizes the surface. We must remark that in [6] the proof is given by explicit constructions of Schottky uniformizations. In this general situation, we only show the existence of them.

In the language of 3-manifolds, the above property can be written as follows. Let $M^{3}$ be the connected sum of $g$ copies of $D^{2} \times S^{1}$, where $D^{2}$ is a closed 2-dimensional disc and $S^{1}$ is the unit circle. We call $M^{3}$ a handlebody of genus $g$. The boundary $S=\partial\left(M^{3}\right)$ is an orientable closed surface of genus $g$. Theorem 1 is equivalent to the following.

Theorem 2. Let $H$ be a group of orientation-preserving homeomorphisms of the boundary $S=\partial\left(M^{3}\right)$, where $M^{3}$ is a handlebody of genus $g$. If $H$ is isomorphic to a dihedral group, then there is an orientation-preserving homeomorphism $f: S \rightarrow S$ so that $f H f^{-1}$ can be extended as a group of orientation-preserving homeomorphisms of $M^{3}$.

The equivalence between both theorems relays on the Nielsen's realization theorem proved by Kerckhoff [7] and Wolpert [12]. Also this is related to the works of B. Zimmermann [13] and A. McCullough, A. Miller and B. Zimmermann [10].

\section{Riemann Matrices Associated to Dihedral Groups}

An object rclated to closed Riemann surfaces are the so called Riemann matrices. These matrices are constructed as follows. Assume $S$ is a closed Riemann surface of genus $g$. A homology basis $\alpha_{1}, \ldots, \alpha_{g}, \beta_{1}, \ldots, \beta_{g}$, is called symplectic if the intersection matrix is

$$
J=\left(\begin{array}{ll}
0 & I \\
-I & 0
\end{array}\right)
$$

where $I$ denotes the identity $g \times g$ matrix. 
Given a symplectic basis $\alpha_{1}, \ldots, \alpha_{g}, \beta_{1}, \ldots, \beta_{g}$, we may find a basis of holomorphic 1-forms on $S$, say $\omega_{1}, \ldots, \omega_{g}$, such that (see [2])

$$
\int_{\alpha_{i}} \omega_{j}=\delta_{i j}
$$

The matrix $Z=\left(\int_{\beta_{i}} \omega_{j}\right)$ is symmetric and with positive definite imaginary part (the Riemann conditions) [2], called the Riemann matrix for $S$ associated to the above symplectic basis.

The Siegel space of genus $g$, denoted by $\mathcal{H}_{g}$, is by definition the space of $g \times g$ matrices with complex coefficients that satisfy to be symmetric and with positive definite imaginary part.

The symplectic group of genus $g$, denoted by $S p_{2 g}(\mathbf{Z})$, consists of all $2 g \times 2 g$ matrices with integer coefficients satisfying the condition $A J A^{t}=J$.

The symplectic group $S p_{2 g}(\mathbf{Z})$ acts on the Siegel space $\mathcal{H}_{q}$ by the rule

$$
\left(\begin{array}{cc}
A & B \\
C & D
\end{array}\right)(Z)=(A+Z C)^{-1}(B+Z D)
$$

Torelli's theorem asserts that the Riemann surface $S$ is determined, up to conformal equivalence, by any of its Riemann matrices.

Let us fix a symplectic basis for the homology of $S$ and let $Z \in \mathcal{H}_{g}$ be the Riemann matrix of $S$ computed in such a basis. Assume now that we have a group $H$ of conformal automorphisms of $S$. Then $H$ induces a faithful action at the level of homology

$$
\theta: H \rightarrow \theta(H) \subset S p_{2 g}(\mathbf{Z})
$$

and induces a faithful action at the level of holomorphic 1-forms

$$
\eta: H \rightarrow \eta(H) \subset G L(g, \mathbf{C}) .
$$

The group $\theta(H)$ fixes the matrix $Z$, that is, $\theta(h)(Z)=Z$ for all $h \in H$. This makes more easy to compute Riemann matrices for Riemann surfaces with large group of automorphisms. Now, if the representation $\theta(H)$ is more or less easy, then we are able to compute explicitly the fixed points of it in the Siegel space.

The condition for the group $H$ to be of Schottky type is equivalent to the existence of some symplectic basis of $S$, say $\alpha_{1}, \ldots, \alpha_{g}, \beta_{1}, \ldots, \beta_{g}$, such that the action of $H$ at the level of homology keeps invariant the submodule 
generated by the loops $\alpha_{1}, \ldots, \alpha_{g}$. In particular, the representation $\theta(H)$ in the symplectic group is of the form

$$
\theta(h)=\left(\begin{array}{ll}
A_{h} & B_{h} \\
0 & C_{h}
\end{array}\right), \quad \text { for each } h \in H
$$

A matrix of the form

$$
M=\left(\begin{array}{cc}
A & B \\
0 & C
\end{array}\right)
$$

belongs to the symplectic group if and only if $B A^{t}$ is symmetric and $C=$ $\left(A^{-1}\right)^{t}$.

In particular, theorem 1 and the above observations give us the following

Theorem 3. Let $H=\left\langle\tau, \sigma: \tau^{n}=\sigma^{2}=(\tau \sigma)^{2}=1\right\rangle$ be a dihedral group of order $2 n$, acting as group of conformal automorphisms of a closed Riemann surface $S$ of genus $g \geq 1$. Then there is a symplectic basis for the homology of $S$ so that

$$
\theta(\tau)=\left(\begin{array}{cr}
x_{1} & x_{2} \\
0 & \left(x_{1}^{-1}\right)^{t}
\end{array}\right) \quad \theta(\sigma)=\left(\begin{array}{lr}
y_{1} & y_{2} \\
0 & \left(y_{1}^{-1}\right)^{t}
\end{array}\right)
$$

such that

(i) $x_{1}^{n}=y_{1}^{2}=\left(x_{1} y_{1}\right)^{2}=1$, that is, $x_{1}$ and $y_{1}$ gives a (faithful) representation of $H$ in $S L(g, \mathbf{Z})$;

(ii) $x_{2} x_{1}^{t}$ and $y_{2} y_{1}^{t}$ are both symmetric;

(iii) $x_{1} y_{1}\left(x_{1} y_{2}+x_{2}\left(y_{1}^{-1}\right)^{t}\right)=-\left(x_{1} y_{2}+x_{2}\left(y_{1}^{-1}\right)^{t}\right)\left(x_{1}^{-1}\right)^{t}\left(y_{1}^{-1}\right)^{t}$.

The above asserts that, for dihedral groups of conformal automorphisms, the computations of Riemann matrices involves only the computations of faithful representations of dihedral groups on $S L(g, \mathbf{Z})$. In the next section we provide some explicit examples of dihedral actions. For these dihedral actions, there exist symplectic basis as in the above theorem with the extra property that $x_{2}=y_{2}=0$ (see for example [14]). 


\section{Examples of Dihedral Actions}

In this section, we consider closed Riemann surfaces with certain type of dihedral groups of conformal automorphisms which are related to certain graphs called prisms and pyramids. Rienann matrices for this class of dihedral groups have been studied in [14]. Let us start with the following result, which is consequence of the results of J.F.X. Ries in [11] and [1].

Theorem 4. Let $h: S \rightarrow S$ be a conformal automorphism of order $n$ of a closed Riemann surface of genus $g \geq 2$. Assume we are in one of the following two situations:

(1) $n=g$ and every non-trivial power of $h$ has exactly two fixed points with opposite rotation numbers:

(2) $n=g-1$ and no non-trivial power of h has fixed points.

Then there is a conformal involution $j: S \rightarrow S$ such that the group generated by $h$ and $j$ is a dihedral group of order $2 n$. Moreover, the topological action is totally determined by the group of symmetries of special graphs in $\mathbf{R}^{3}$ called $g$-pyramids and $g$-prisms.

We give a different proof, using Schottky uniformizations, of theorcm 4 . We decompose it into lemmas 1 and 2 below, which are proven in section 5 . The proves are given by explicit construction of Schottky uniformizations. We also construct, at the end of this section, explicit Schottky groups uniformizing Riemann surfaces that reflects the groups of automorphisms of $g$-prisms.

Let us start with some notations. Let us denote by $\mathbf{R}^{3}$ the three dimensional euclidean space. We identify this space with $\mathbf{C} \times \mathbf{R}$, where $\mathbf{C}$ is the complex plane. In this way, we can think of the points in $\mathbf{R}^{3}$ as pairs $(z, t)$ where $z \in \mathbf{C}$ and $t \in \mathbf{R}$. The one-point compactification of $\mathbf{R}^{3}$ is the 3-sphere $S^{3}=\mathbf{R}^{3} \cup\{\infty\}$ containing the Riemann sphere $\hat{\mathbf{C}}$.

Fix an integer number $g \geq 2$, and denote by $S^{1} \subset \mathbf{C}$ the unit circle. Set $v_{k}=\left(e^{2 \pi(k-1) r / g}, 0\right)$, where $k=1,2, \ldots, g$, and $w=(0,0)$. Denote by $e_{j}$ the arc in $S^{1} \times\{0\}$ joining the vertices $v_{j}$ and $v_{j+1}$. Set $f_{k}=\left\{t v_{k} ; t \in[0,1]\right\}$, for $j, k=1, \ldots, g$. The graph constructed in $S^{3}$ with vertices $v_{1}, \ldots, v_{g}, w$, and edges $e_{1}, \ldots, e_{g}, f_{1}, \ldots, f_{g}$, is called a $g$-pyramid and denoted by $P_{g}$. 
Set $T(z, t)=(2 z, 2 t)$ and let $C_{g} \subset S^{3}$ be the graph constructed as follows. The vertices are $u_{r}=\left(2 e^{2 \pi(r-1) i /(g-1)}, 0\right)$ and $w_{r}=\left(\frac{1}{2} e^{2 \pi(r-1) i /(g-1)}, 0\right)$, for $r=1, \ldots, g-1$. The edges of $C_{g}$ are given by the arcs $j_{r} \subset T\left(S^{1}\right)$ connecting the vertices $u_{r}$ and $u_{r+1}$, the $\operatorname{arcs} k_{r}=T^{-1}\left(e_{r}\right)$, and $l_{r}=$ $\left\{t u_{r}+(1-t) w_{r} ; t \in[0,1]\right\}$, for $r=1, \ldots, g-1$. We call $C_{g}$ a $g-$ prism.

Let us consider the conformal automorphisms of $S^{3}$ given by $b(z, t)=$ $(\bar{z},-t), c(z, t)=(1 / \bar{z},-t)$ and $a_{k}(z, t)=\left(e^{2 \pi i / k} z, t\right)$, where $k$ is a nonnegative integer.

It is a well known fact (see [3]) that the group of symmetries of the graph $P_{g}$ has the following description.

(1) If $g \geq 4$, then it is isomorphic to the dihedral group of order $2 g$. This group is generated by the transformations $a_{g}$ and $b$.

(2) If $g=3$, then it is isomorphic to the symmetric group in four letters $S_{4}$, generated by $a_{3}, b$ and an extra element $d$ of order 4 .

Denote in both cases the subgroup generated by $a_{g}$ and $b$ as $D_{g}$.

The group of symmetries of the graph $C_{g}$ has the following description.

(1) If $g \neq 5$, then it is the direct $\operatorname{sum}$ of $\mathbf{Z} / 2 \mathbf{Z}$ (generated by $c$ ) and a dihedral group of order $2(g-1)$ (generated by $a_{g-1}$ and $b$ ).

(2) If $g=5$, then there is an extra symmetry $d$, of order two, such that $a_{g-1}, b, c$ and $d$ generate the symmetry group of $P_{5}$. This group is isomorphic to the symmetric group $\mathcal{S}_{\mathbb{A}}$ in four letters.

We denote the group of symmetries of $C_{g}$ by $F_{g}$. Its subgroup, generated by $a_{g-1}$ and $b$, is a dihedral group of order $2(g-1)$ and we denote it by $D_{g-1}^{\prime}$.

We can construct a tubular neigliborhood $V_{g}$ (respectively, $W_{g}$ ) of $P_{g}$ (respectively, $C_{g}$ ), which is invariant under the action of $D_{g}$ (respectively, $F_{g}$ ). In this case, $V_{g}$ (respectively, $W_{g}$ ) is a compact 3 -manifold homeonorphic to a handlebody of genus $g$. The boundary $S_{g}=\partial V_{g}$ (respectively, $R_{g}=\partial W_{g}$ ) is a closed orientable surface of genus $g$. The group $D_{g}$ (respectively, $F_{g}$ ) acts on both $V_{g}$ and $S_{g}$ (respectively, $W_{g}$ and $R_{g}$ ) as a group of orientation preserving homeomorphisms. The Nielsen realization theorem [7] asserts that

(i) we can give to $S_{g}$ (respectively, $R_{q}$ ) a Riemann surface structure for which $D_{g}$ (respectively, $F_{g}$ ) acts as a group of conformal automorphisms, and 
(ii) we can give to $V_{g}$ (respectively, $W_{g}$ ) a hyperbolic structure for which $D_{g}$ (respectively, $F_{g}$ ) acts as group of isometries.

Let $S$ be a closed Riemann surface of genus $g \geq 2$. Assume $H$ is a group of conformal automorphisms of $S$. We say that $H$ is of pyramidal type if:

(1) there is an orientation preserving homeomorphism $\Phi: S \rightarrow S_{g}$ such that, $\Phi h \Phi^{-1} \in D_{g}$ for all $h \in H$ and

(2) $\Psi: H \rightarrow D_{g}$, defined by $\Psi(h)=\Phi / \iota \Phi^{-1}$, is an isomorphism of groups.

If $h: S \rightarrow S$ is a conformal automorphism of some Ricmann surface $S$, and $p \in S$ is fixed point of it, then we can define a rotation number $\alpha(h, p) \in(-\pi, \pi]$ so that, in local coordinates $z$ with $z(p)=0$, we have $h(z)=e^{\alpha(h, p) i} z$.

Lemma 1. Let $S$ be a closed Riemann surface of genus $g \geq 2$. Assume that there is a conformal automorphism $h: S \rightarrow S$, of order $g$, having exactly two fixed points, say $p$ and $q$. If

(1) $\alpha(h, p)=-\alpha(h, q)$ for $g \geq 3$, and

(2) every non-trivial power of h has exactly two fixed point (that is, $p$ and $q)$,

then there is a conformal involution $j: S \rightarrow S$ so that the group generated by $h$ and $j$ is of pyramidal type.

Let $S$ be a closed Riemann surface of genus $g \geq 2$. Assume $H$ is a group of conformal automorphisms of $S$. We say that $H$ is of prism type if:

(1) there is a preserving orientation homeomorphism $\Phi: S \rightarrow R_{g}$ so that, $\Phi h \Phi^{-1} \in D_{g-1}^{\prime}$ for all $h \in H$ and

(2) $\Psi: H \rightarrow D_{g-1}^{\prime}$, defined by $\Psi(h)=\Phi / h \Phi^{-1}$, is an isomorphism of groups.

Lemma 2. Let $S$ be a closed Riemann surface of genus $g \geq 2$. Assume that there is a conformal automorphism $h: S \rightarrow S$ of order $g-1$. If no non-trivial power of it has fixed points, then there is a conformal involution $j: S \rightarrow S$ so that the group generated by $h$ and $j$ is of prism type. 


\section{The examples}

We proceed to construct Schottky uniformizations of Riemann surfaces of genus $g$ admitting a conformal group topologically equivalent to $F_{g}$.

\section{1.- Case $g \neq 5$}

We have that $F=F_{g}$ has generators $a, b$ and $c$, with relations

$$
a^{g-1}=b^{2}=c^{2}=(a b)^{2}=c a c a^{-1}=(c b)^{2}=I .
$$

Let $P: R \rightarrow R / F$ the holomorphic branched covering induced by the action of $F$ on $R$. We have that $R / F$ has five branch values of order two. They are as follows

(1) two of them, say $p$ and $q$, are projections of two fixed points of $b$;

(2) one, say $r$, is projection of a fixed point of $c$;

(3) one, say $s$, is projection of a fixed point of $a b$; and

(4) one, say $t$, is projection of a fixed point of $c a b$.

We can uniformize the surface $R / F$ by a Kleinian group $K$ constructed from the first Maskit-Klein combination theorem [8] with the finite groups $K_{1}=\left\langle J, T ; J^{2}=T^{2}=(J T)^{2}=I\right\rangle$ and $K_{2}=\left\langle N, N^{2}=I\right\rangle$.

A fundamental domain $D$ of $K$ is drawn in figure 1. We also assume that the fixed points of $N$ are projected onto the points $p$ and $q$, the fixed points of $J$ and $T$ contained in the boundary of $D$ are projected onto the points $r$ and $s$, respectively, and the fixed point of $J T$ is projected onto $t$.
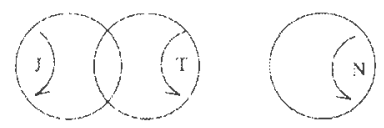

D.

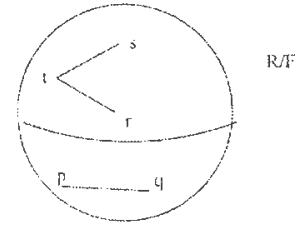


The existence of such a group is due to (i) quasiconformal deformation theory and (ii) the fact that we can construct a particular group $K_{0}$ as desired (the quotient $\Omega\left(K_{0}\right) / K_{0}$ does not need to be $R / F$ ).

We have the following properties:

(5) $K$ is free product of $K_{1}$ and $K_{2}$;

(6) $K$ is geometrically finite;

(7) the region of discontinuity $\Omega$ of $K$ is connected;

(8) every non-loxodromic element of $K$ is conjugate to either $J, T, J T$ or $N$.

We have a natural surjective homomorphism $\Phi: K \rightarrow F$ defined by $\Phi(J)=c, \Phi(T)=a b$ and $\Phi(N)=b$. This homomorphism is constructed by the lifting of a suitable base for the fundamental group of $R / F-B$, where $B=\{p, q, r, s, t\}$.

The kernel of $\Phi$ is the smallest normal subgroup of $K$ containing the transformations $A^{g-1},(J N)^{2}$ and $J A J A^{-1}$, where $A=T N$. Let us denote this subgroup by $G$.

We have that $G$ is torsion-free since neither $J, T, J T$ nor $N$ belong to it.

Since $G$ has finite index in $K$, we have that its region of discontinuity is also $\Omega$, and that $G$ is geometrically finite.

As a consequence of [9], we have that $G$ is in fact a Schottky group.

The Riemann-Hurwitz formula and the fact that $K / G$ has order $4(g-1)$, imply that the genus of $G$ is $g$.

The construction asserts that there is a conformal equivalence $f: \Omega / G \rightarrow$ $R$ such that $f(K / G) f^{-1}=F$.

\section{2.- Case $g=5$}

The group $F=F_{5}$ has generators $a$ and $b$ with relations

$$
a^{4}=b^{2}=(b a)^{3}=I .
$$

Let $P: R \rightarrow R / F$ the holomorphic branched covering induced by the action of $F$ on $R$. We have that $R / F$ has two brancli values of order two and two of order three. They are 
(1) two of them, say $p$ and $q$, are projections of two fixed points of $b$; and

(2) the other two, say $r$ and $s$, is projection of a fixed point of $b a$.

We can uniformize the surface $R / F$ by a Kleinian group $K$ constructed from the first Maskit-Klein combination theorem [8] with the finite groups $K_{1}=\left\langle T ; T^{3}=I>\right.$ and $K_{2}=\left\langle N, N^{2}=I\right\rangle$. A fundamental domain $D$ of $K$ is drawn in figure 2. We also assume that the fixed points of $N$ are projected onto the points $p$ and $q$, and the fixed points $T$ are projected onto the points $r$ and $s$.

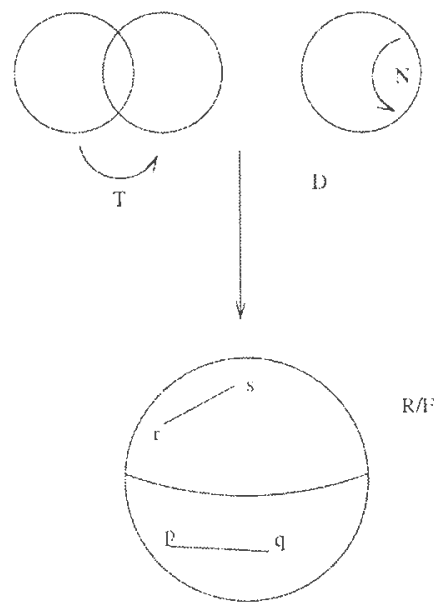

FiaUR: 2

We have the following propertics:

(3) $K$ is free product of $K_{1}$ and $K_{2}$;

(4) $K$ is geometrically finite;

(5) the region of discontinuity of $K$, say $\Omega$, is connected;

(6) every non-loxodronic element of $K$ is conjugate to either $N, T$ or $T^{2}$. 
We have a natural surjective homomorphism $\Phi: K \rightarrow F$ defined by $\Phi(N)=b, \Phi(T)=b a$. This homomorphism is constructed by the lifting of a suitable base for the fundamental group of $R / F-B$, where $B=\{p, q, r, s\}$. The kernel of $\Phi$ is the smallest normal subgroup of $K$ containing the transformation $A^{4}$, where $A=N T$. Let us denote this subgroup by $G$ and denote by $B$ the transformation $N$.

We have that $G$ is torsion-free since neither $T, T^{2}$ nor $N$ belong to it. Since $G$ has finite index in $K$, we have that its region of discontinuity is also $\Omega$, and that $G$ is geometrically finite.

As a consequence of [9], we have that $G$ is in fact a Schottky group.

The Riemann-Hurwitz formula together to the fact that $K / G$ has order 24 assert that the genus of $G$ is 5 .

The construction asserts that there is a conformal equivalence $f: \Omega / G \rightarrow$ $R$ such that $f(K / G) f^{-1}=F$.

\section{Proof of Theorem 1}

Assume we are able to construct a collection of pairwise disjoint simple loops on the surface $S$ such that they cut off $S$ into planar surfaces. Then we can find a sub-collection of these loops such that they do not disconnect $S$ but they cut off $S$ into a planar surfacc (a $2 g$-connected surface). The subcollection define a Schottky uniformization of $S$ (take the normal subgroup generated by these loops in the fundamental group of $S$ ).

If such a collection of loops has the extra property that it is invariant under the action of $H$, then basic lifting criterion asserts that $H$ lifts to the Schottky uniformization defined by them.

\section{Construction of a Collection of Simple Loops}

Let us consider a presentation for the group $H$ of the form $H=\left\langle\tau, \sigma: \tau^{n}=\right.$ $\left.\sigma^{2}=(\tau \sigma)^{2}=1\right\rangle$.

We denote by $P: S \rightarrow R=S /\langle\tau\rangle$ the canonical (branched) Galois covering induced by the cyclic group $\langle\tau\rangle$ of order $n$ generated by $\tau$.

Since $\sigma$ has order two and conjugate $\langle\tau\rangle$ in itself, we have the existence of a conformal automorphism $\widehat{\sigma}: R \rightarrow R$ satisfying $P \sigma=\widehat{\sigma} P$. All the liftings of $\widehat{\sigma}$ are the $n$ involutions $\tau^{k} \sigma, k=0, \ldots, n-1$.

The topological action of the involution $\hat{\sigma}: R \rightarrow R$ is well understand and it is shown in figure 3 . We have two possibilities:

(1) $\operatorname{Fix}(\widehat{\sigma})$ is non-empty; or

(2) $\operatorname{Fix}(\widehat{\sigma})$ is cmpty. 

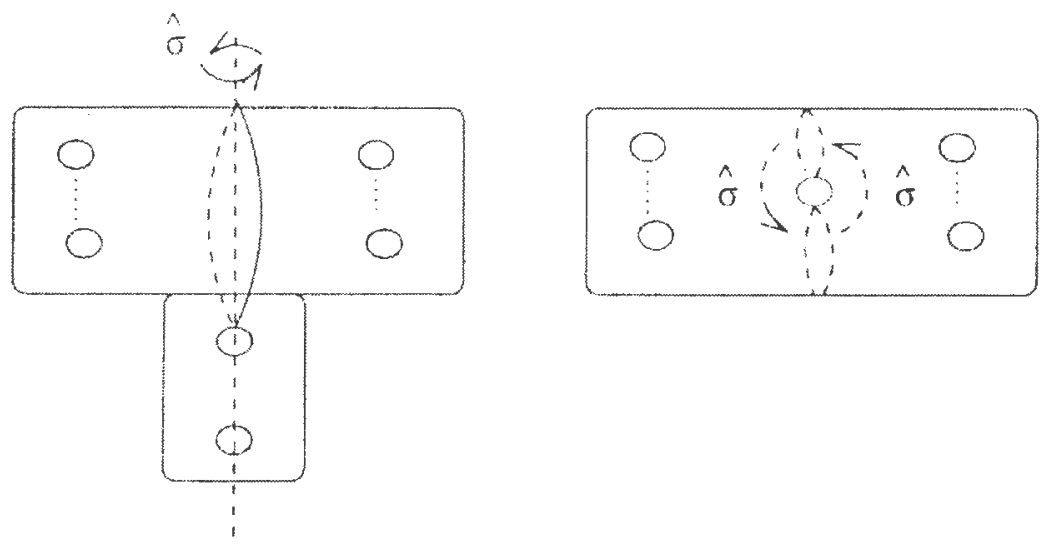

\section{Figure 3. The topological action of $\widehat{\sigma}$}

We proceed to show the existence of a collection of loops as desired in each situation. We must remark that a branch value of $P: S \rightarrow R$ cannot be a fixed point of $\widehat{\sigma}$. In fact, if this is the case, then there is a point $y \in S$ which is fixed by some non-trivial power of $\tau$ and fixed by some element of the form $\tau^{\alpha} \sigma$, where $a \in\{0,1,2\}$. In any case, it will asserts that the stabilizer of $y$ in $H$ is not cyclic, a contradiction.

Case 1: Let us assume Fix $(\widehat{\sigma}) \neq \emptyset$. In this case, we proceed to draw a collection of simple loops as shown in figure 4 . Observe that if no nontrivial power of $\tau$ has fixed points, then the loops $\eta_{j}$ and the regions $A_{j}$ are not there. 


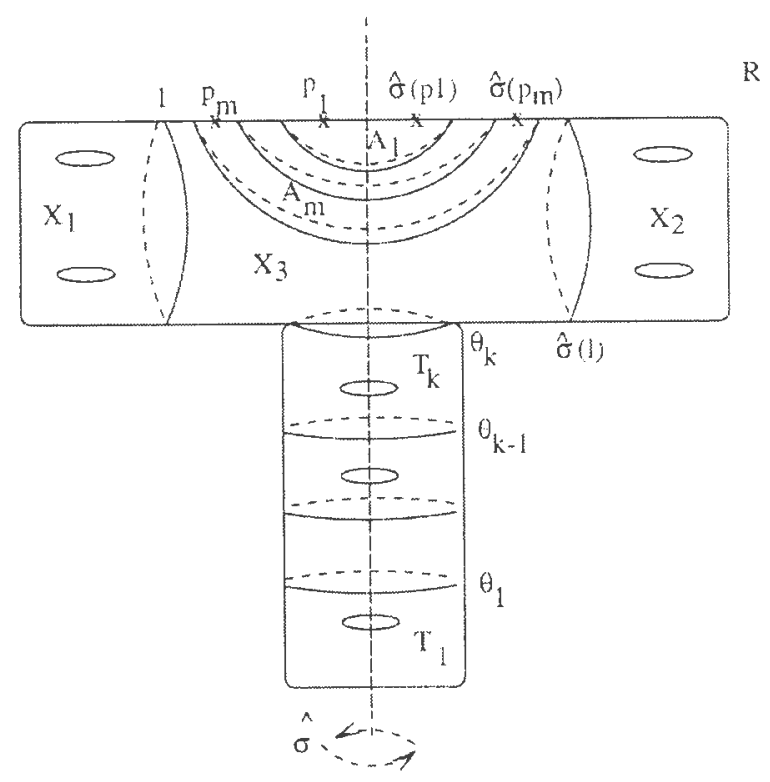

Figure 4. A collection of simple loops on $R$

Each of these loops lifts to exactly $n$ simple loops, permuted by $\tau$. The reason is that $l, \hat{\sigma}(l), \theta_{1}, \ldots, \theta_{k}$ are product of commutators. The loop $\eta_{1}$ bounds a disc containing only the branch values $p_{1}$ and $\widehat{\sigma}\left(p_{1}\right)$. The rotation numbers at these points are the same but with opposite sign (this because $\sigma$ conjugates each $\tau^{a}$ into $\tau^{-a}$. It follows that $\eta_{1}$ lifts to exactly $n$ loops. In similar fashion we see that the other loops $\eta_{2}, \ldots, \eta_{m}$, each one lifts to exactly $n$ loops.

Let us consider a connected component $Y_{1}$ of $P^{-1}\left(X_{1}\right)$, where $X_{1}$ is the subsurface of $R$ bounded by the loop $l$ as shown in figure 3. Since the loop $l$ lifts to exactly $n$ loops, we have that such a component is a closed Riemann surface with $r$ pairwise disjoint discs removed, on which a cyclic group of order $r$ acts free fixed points and permutes cyclically the boundary components. As a consequence of the results in [4], we are able to find a collection of pairwise disjoint simple loops on $Y_{1}$ that is invariant under the cyclic group and cut off $Y_{1}$ into genus zero surfaces. We consider on each translate of $Y_{1}$ under the group $H$ the translates of the loops on $Y_{1}$. The action on the connected components of $P^{-1}\left(\widehat{\sigma}\left(X_{1}\right)\right)$ is obtained by conjugating the above by $\sigma$. 
A connected component of $P^{-1}\left(X_{3}\right)$, where $X_{3}$ is the subsurface bounded by the simple loops $\theta_{k}, l \eta_{m}$ and $\widehat{\sigma}(l)$ is homeomorphic to $X_{3}$. This because each of the boundary loops lifts to exactly $n$ simple loops.

Let $B_{1}$ be a connected component of $P^{-1}\left(A_{1}\right)$. This surface is precisely invariant under a cyclic subgroup of $\langle\tau\rangle$ of order $r_{1}$ (exactly the order of branching of the two points enclosed by $\eta_{1}$, and a involution $\tau^{a} \sigma$. Since the boundary loop $\eta_{1}$ lifts to exactly $n$ loops, we have that by gluing discs to the boundaries of both $A_{1}$ and $B_{1}$ we do not add branching. We may apply Riemann-Hurwitz's formula to obtain that $B_{1}$ is a genus zero surface with exactly $r_{1}$ boundary loops (permuted cyclically by the above cyclic subgroup).

For the subsurface $A_{j}$ bounded by the loops $\eta_{j-1}$ and $\eta_{j}$ the situation is similar to the above case.

Let $Z_{1}$ be a connected component of $P^{-1}\left(T_{1}\right)$. Again, the fact that $\theta_{1}$ lifts to exactly $n$ loops, the stabilizer of $Z_{1}$ is a dihedral group generated by some power of $\tau$ and some element of order two of the form $\tau^{a} \sigma$. By gluing discs to the boundaries of $Z_{1}$ and $T_{1}$ we only add one branched value of order two on the surface obtained from $T_{1}$. Each gluing disc to the component $Z_{1}$ produces exactly one fixed point of a conjugate of the element $\tau^{a} \sigma$. Applying Riemann-Hurwitz's formula, we obtain that $Z_{1}$ is a genus one Riemann surface admitting a dihedral action. This dihedral action in a closed torus is generated by a rotation (acting free fixed points) and an involution that fixes exactly 4 points. There is a collection of pairwise disjoint simple loops on $Z_{1}$, invariant under the dihedral action, and cutting off this torus with holes into genus zero surfaces. We translate these loops by the action of the cyclic group generated by $\tau$ to the other components of $P^{-1}\left(T_{1}\right)$.

For the subsurfaces $T_{j}$ we proceed in similar fashion.

Putting all together, we obtain a collection of pairwise disjoint simple loops on $S$ that cut off it into genus zero surfaces and it is invariant under the action of $H$.

Case 2: Let us assume $F i x(\widehat{\sigma})=\emptyset$. In this case, we proceed to draw a simple loop $\gamma$ that is invariant under $\widehat{\sigma}$, disjoint from the branched values of the (branched) covering $P: S \rightarrow R$ and homologically non-trivial as shown in figure 5 . If we show that the loop $\gamma$ lifts to exactly $n$ loops, then we can procced as in the above case (think of such a loop as the fixed point of $\widehat{\sigma}$ enclosed by the loops $\eta_{1}$ in that situation). 


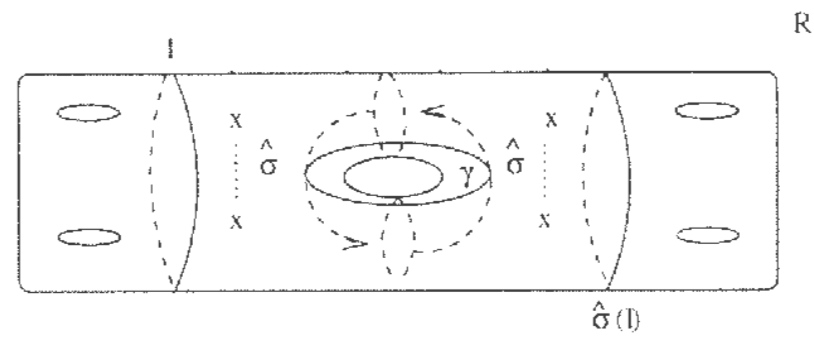

Figure 5. A simple loop $\%$ on $R$

To show the lifting property of $\gamma$, we consider a comnected component $\eta$ of $P^{-1}(\gamma)$. If our claim is not true, then the stabilizer in $H$ of $\eta$ is a dihedral group acting free fixed points. But this will asserts that a dihedral group is a surjective homomorphism image of $\mathbf{Z}$, a contradiction.

Let us consider the collection of loops as showit in figure 6 . Now we can procecd as in case 1 to construct the desired collection of loops. The proof of theorem 1 is now finished.

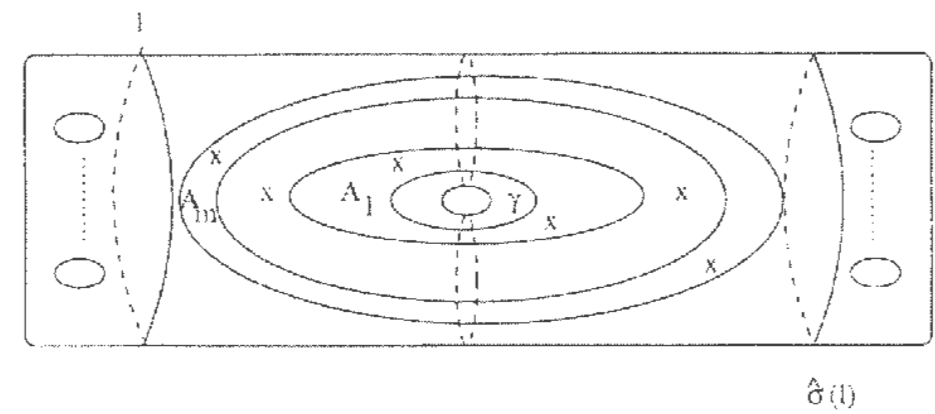

Figure 6. A collection of simple loops on $R$ 


\section{Proof of Theorem 4}

In this section, we first prove lemmas 1 and 2. Theorem 4 is just combination of both lemmas. The prove of both lenmas are constructive and givo explicit Schottky uniformizations.

\subsection{Proof of Lemma 1}

Let us consider the branclied sovering $P: S \rightarrow R=S / H$, where $H$ is the cyclic group gencrated by $h$. We may assume that $h$ satisfies the following minimal condition

$$
0<\alpha(h, p) \leq \alpha\left(h^{r}, p\right), \quad r=1,2, \ldots, g-1 .
$$

Set $u=P(p), v=P(q), \tilde{S}=S-\{p, q\}$ and $\tilde{R}=R-\{u, v\}$. Wo still denoting by $P$ the induced covering obtained by restriction of the above one. The Ricmann-Hurwitz formula asserts that $R$ is a genus one surface.

Consider oriented simple loops $\alpha, \beta, \delta$ and $\eta$, throngh a point $x \in \tilde{R}$ as shown in figure 7 .

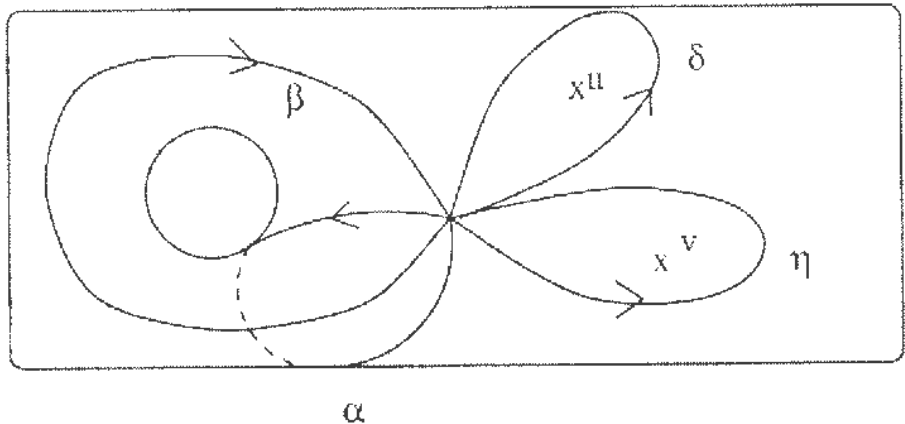

$\mathrm{R}$

FIGURE 7 
The fundamental group of $\tilde{R}$, based at $x$, is generated by $\alpha, \beta, \delta$ and $\eta$, with relation $[\alpha, \beta] \eta \delta=1$, where $[\alpha, \beta]$ denotes the commutator $\beta^{-1} \alpha^{-1} \beta \alpha$.

Let $y \in \tilde{S}$ be such that $P(y)=x$. We have a natural surjective homomorphism

$$
\Psi_{g}: \Pi_{1}(\tilde{R}, x) \rightarrow H,
$$

defined by lifting loops, based at $x$, with starting point $y$.

Since the group $H$ is Abelian and $\alpha(h, p)=-\alpha(h, q)$ (if order of $h$ is bigger than two), we have that

$$
\Psi_{g}(\eta)=h^{r}, \quad \Psi_{g}(\delta)=h^{-r}, \quad \Psi_{g}([\alpha, \beta])=1,
$$

where $h^{r}$ generates $H$. As a consequence of proposition 6 of [5], wo may assume that $\Psi_{g}(\beta)=1$ and $\Psi_{g}(\alpha)=h$.

By quasiconformal deformation theory, we can find a (branched) holomorphic covering $Q: \Omega \rightarrow R$ such that:

(1) its covering group is a Kleinian group $\tilde{G}$ with $\Omega$ as its region of discontinuity;

(2) $\tilde{G}$ is a cyclic normal extension of a Schottky group of genus one, freely generated by a loxodromic element $L$ and an clliptic element $E$ of order $g$;

(3) the fixed points of $E$ are projected by $Q$ onto the points $u$ and $v$, respectively;

(4) a fundamental domain $D$ (see figure 8 ) for $\tilde{G}$ is bounded by three disjoint simple loops $\gamma^{+}, \gamma^{-}$and $\gamma$, satisfying

(4.1) $L\left(\gamma^{-}\right)=\gamma^{+}, E(\gamma)=\gamma$,

(4.2) both fixed poirits of $E$ belong to $\gamma$,

(4.3) the loops $\gamma^{ \pm}$are projected onto the loop $\beta$,

(4.4) the loop $\gamma$ is projected onto a simple path connecting $u$ and $v$ and disjoint from $\alpha$. 


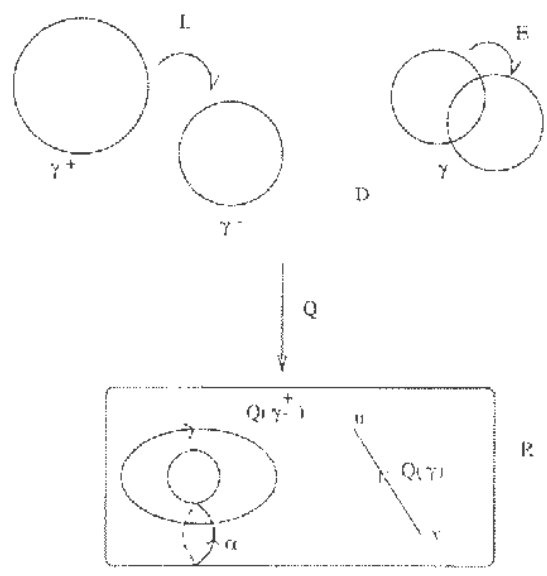

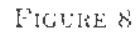

Consider the subgroup $G$ generated by the trans formations $L_{k}=E^{k} L E^{-k}$, $k=0,1, \ldots, g-1$. We have that $G$ is a normal subgroup of $\tilde{G}$ of index $g$ and $\bar{G} / G$ is isomorphic to the cyclic group $H$.

The normality of $G$ asserts that $\Omega$ is also the region of discontinuity of $G$.

By the choice of the loops $\alpha, \beta, \delta$ and $\eta$, wo have that the coverings $P$ : $S \rightarrow R$ and the natural covering $\Omega / G \rightarrow R$, induced by $Q$, are equivalent. The automorphism $h: S \rightarrow S$ corresponds to the automorphism induced by $F$.

$\mathrm{U}_{\mathrm{p}}$ to conjugation, we niny assume that

$$
E=\left(\begin{array}{cc}
e^{\pi i / g} & 0 \\
0 & e^{-\pi i / g}
\end{array}\right),
$$

and one fixed of $L$ is 1 . Denote by $r$ the other fixed point of $L$. Then $r \in \mathrm{C}-\{0,1\}$. Let us consider the transformation

$$
J=\left(\begin{array}{cc}
0 & r \\
1 & 0
\end{array}\right) .
$$

Direct conputations show that 
(1) $J^{2}=I$

(2) $J E J=E^{-1}$

(3) $J L J=L^{-1}$

Let $K$ be the group generated by $E, L$ and $J$. We have that $K$ is a finite normal extension of

(1) $\tilde{G}$ of index two, and

(2) $G$ of index $2 g$.

Moreover, $K / G$ is isomorphic to a dihedral group of order $2 g$.

The transformation $J$ induces an automorphism $j: S \rightarrow S$ of order two. We have then that $j$ and $h$ generates a group $\mathcal{D}$ isomorphic to a dihedral group of order $2 g$.

By construction, it is not hard to see that the topological action of $\mathcal{D}$ on $S$ is topologically the same as the one given by $D_{g}$ on $S_{g}$.

We must note that the automorphism $j$ corresponds to the automorphism $a b \in D_{g}$ and $h$ (with the minimal rotation condition) corresponds to $a \in D_{g}$. We have proved lemma 1 .

Remark. Another approach to this theorem is the following. Let us denote by

(1) $H$ the cyclic group generated by $h: S \rightarrow S$;

(2) $R$ the quotient Riemann surface obtained by the action of $H$ on $S$;

(3) $P: S \rightarrow R$ the natural holomorphic branched covering induced by $H$.

By the formula of Riemann-Hurwitz and the assumptions on the fixed points of the elements of $H$, we have that $R$ is a torus with exactly two branch values of order $g$. Set $x=P(p)$ and $y=P(q)$ these two branch values.

Since $x \neq y$, there is a unique conformal involution $J: R \rightarrow R$ such that $J(x)=y$ and having exactly four fixed points. As a consequence of our hypothesis on the rotation numbers, we can lift $J$ to a conformal automorphism of $S$. 
If $;: S \rightarrow S$ denotes on of such liftings, that is, $P \cdot j=J P$, then $j(p)=q$. In fact, sinco $J(x)=y$. we must have that $j(p)$ and $q$ are $H$ equivalent. B311. $q$ is a fixed point of cvery clement in $H$, that is, $j(p)=q$.

'The fact that $J$ has fixed points implies that there is a point $s \in S-$ $\{p, q\}$ with $j(s)=h^{r}(s)$. In particular. $h^{-r} j$ fixos the point $s$. Since $h^{-r} j$ is again a lifting of $J$, we nady assune that is the casc for $j$.

The property $J^{2}=I$ implics that $j^{2}$ belongs to $H$ and, in particnlar, by our hypothesis on $I I$ ank the above fact that $j^{2} \in H$ fixes $s \notin\{p, q\}$, we have $j^{2}=I$.

We also have that $j h j=h^{t}$, where $t \in\{1, \ldots, g-1\}$ and $(g, t)=1$. By looking at the rotation numbers, we have the following:

$$
-\operatorname{ta}(h, \varphi)=\operatorname{ta}(h, p)=\alpha\left(h^{l}, p\right)=\alpha(h h j, p)=\alpha(h, j(p))=\alpha(h, q) .
$$

It follows that $t=-1$ and, in particular, $j$ and $h$ generate a dihedral group of order $2 g$.

\subsection{Proof of Lemma 2}

Let us consider the unbrancled covering $P: S \rightarrow R=S / H$, where $H$ is the cyclic group gonerated by $h$. The Riemann-Hurwitz formula asserts that $R$ is a genus two surface.

Consider oriented simple loops $\alpha_{1}, \alpha_{2}, \beta_{1}$ and $\beta_{2}$, through a point $x \in R$ as shown in figure 9 .

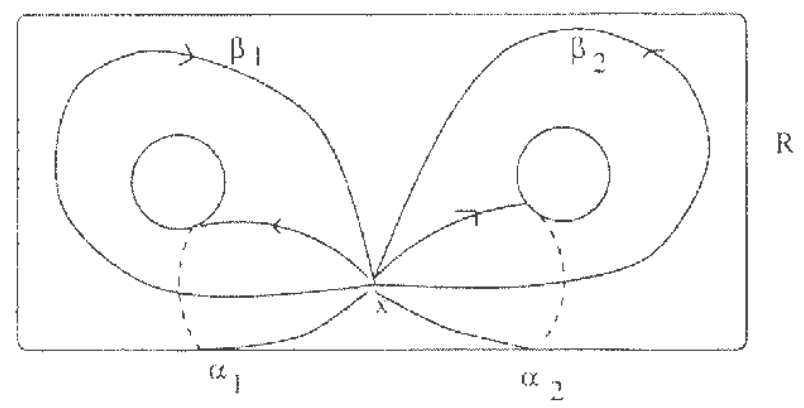

FIGURE ? 
The fundamental group of $R$, based at $x$, has generators $\alpha_{1}, \alpha_{2}, \beta_{1}$ and $\beta_{2}$, and relation $\left[\alpha_{1}, \beta_{1}\right]\left[\alpha_{2}, \beta_{2}\right]=1$, where $\left[\alpha_{i}, \beta_{i}\right]=\beta_{i}^{-1} \alpha_{i}^{-1} \beta_{i} \alpha_{i}$.

If $y \in S$ is such that $P(y)=x$, then wo lave a natural surjective homomorphism

$$
\Psi_{g}: \Pi_{1}(R, x) \rightarrow H
$$

defined by lifting loops at $y$.

Since the group $H$ is Abelian, we have that

$$
\Psi_{g}\left(\left[\alpha_{i}, \beta_{i}\right]\right)=1
$$

By proposition 6 of [5], we may assume that $\Psi_{g}\left(\beta_{1}\right)=\Psi_{g}\left(\beta_{2}\right)=$ $\Psi_{g}\left(\alpha_{2}\right)=1$ and $\Psi_{g}\left(\alpha_{1}\right)=h$.

By quasiconformal deformation theory, we can find a Kleinian group $\tilde{G}$ (a cyclic normal extension of a Schottky group of genus two), with region of discontinuity $\Omega$, generated by loxodromic elements $A, L$ and an elliptic element $E$ of order $g-1$, such that

(1) $E A=A E$,

(2) there is a holomorphic covering $Q: \Omega \rightarrow R$ with $\tilde{G}$ as covering group, and

(3) $\tilde{G}$ has a fundamental domain $D$ (as shown in figure 10) bounded by four $\operatorname{arcs} \eta^{ \pm}, \delta^{ \pm}$and two curves $\gamma^{ \pm}$, such that

(3.1) $L\left(\gamma^{+}\right)=\gamma^{-}, A\left(\eta^{+}\right)=\eta^{-}, E\left(\delta^{+}\right)=\delta^{-}$.

(3.2) The loop $\gamma^{ \pm}$is projected onto a simple loop free homotopic to $\alpha_{2}$.

(3.3) The arc $\eta^{ \pm}$is projected to a simple loop free homotopic to $\alpha_{1}$.

(3.4) The arc $\delta^{ \pm}$is projected to a simple loop free homotopic to $\beta_{1}$.

(3.5) The loop $\beta_{2}$ can be lifted, in $D$, to a simple path connecting the loops $\gamma^{+}$and $\gamma^{-}$. 


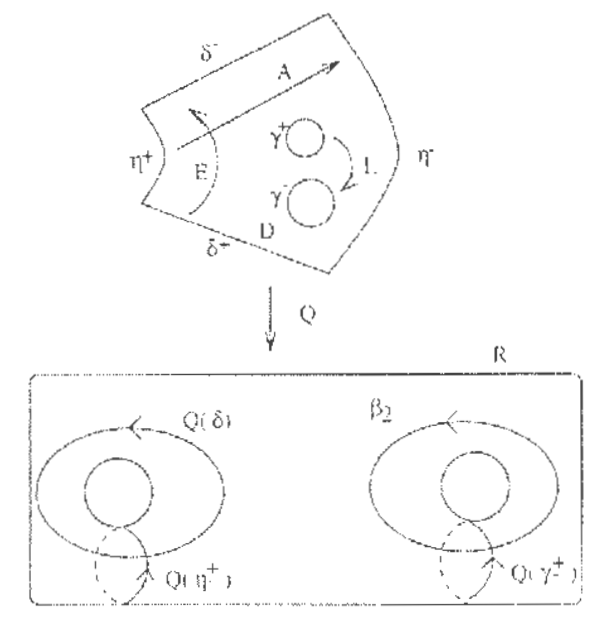

Frowiti 10

Consider the subgroup $G$ generated by the transformations $A$ anc1 $L_{k}=$ $E^{k} L E^{-k} \cdot k=0,1, \ldots, g-2$. We have that $G$ is a normal subgroup of $\tilde{G}$ of index $y-1$ and $\bar{G} / G$ is isomorplice to the eyclic group $H$.

The normality of $G$ asserts that $\Omega$ is also the region of discontinuity of G.

By the choice of the loor $\alpha_{1}, \alpha_{2}, \beta_{1}, \beta_{2}$, we have that the coverings $P$ : $S \rightarrow R$ and the natural covering $\Omega / G \rightarrow R$, induced by $Q$, are equivalent.

The autonorphism $h: S \rightarrow S$ corresponkls to the automorphism induced by $E$.

Up to conjugation, we may assume that

$$
E=\left(\begin{array}{cr}
e^{\pi i /(g-1)} & 0 \\
0 & e^{-\pi i /(q-1)}
\end{array}\right)
$$

and one fixed of $L$ is 1 . Denote by $r$ the other fixed point of $L$. Then $r \in \mathbf{C}-\{0,1\}$. Let us considem the transformation

$$
J=\left(\begin{array}{ll}
0 & r \\
1 & 0
\end{array}\right) .
$$


In particular, the transformation $A$ has the form $A(z)=\lambda z$, for some $\lambda \in \mathbf{C}$ with absolute value different from 0 and 1 . Direct computations show that

(1) $J^{2}=I$

(2) $J E J=E^{-1}$

(3) $J L J=L^{-1}$

(4) $J A J^{-1}=A^{-1}$

Let $K$ be the group generated by $E, A, L$ and $J$. The group $K$ is a finite normal extension of

(1) $\tilde{G}$ of index two,

(2) $G$ of index $2(g-1)$.

Moreover, $K / G$ is isomorphic to the dihedral group of order $2(g-1)$. The automorphism $j: S \rightarrow S$ induced by $J$ is an involution that together $h$ generates a dihedral group.

By the construction, it is not hard to sce that the topological action of this dihedral group on $S$ is the same topological action of $D_{g-1}^{\prime}$ on $R_{g}$. We have proved lemma 2.

Remark. Another proof of this is the following. Let us denote by

(1) $H$ the cyclic group generated by $h: S \rightarrow S$;

(2) $R$ the quotient Riemann surface obtained by the action of $H$ on $S$;

(3) $P: S \rightarrow R$ the natural holomorphic branched covering incluced by $H$.

By the formula of Rienanu-Hurwitz and the assumptions on the no existence of fixed points for the elements of $H$, we have that $R$ is a genus two surfacc.

Let us consider the hyperelliptic involution $J: R \rightarrow R$. By Farkas result in [1], we can lift it to a conformal automorphism of $S$.

Let $j: S \rightarrow S$ be a lifting of $J$, that is, $P j=J P$. Since $J$ has fixed points, we have the existence of a point $s \in S$ with $j(s)=h^{r}(s)$. In 
particular, $h^{-r} j$ fixes the point $s$. Since $h^{-r} j$ is also a lifting of $J$, we may assume that is the case for $j$.

The property $J^{2}=I$ asserts that $j^{2}$ belongs to $H$. But $j^{2}$ fixes $s$. By our hypothesis on the fixed points of the elements of $H$, the above is only possible if $j^{2}=I$.

Let us now consider the lifting of $J$ given by $h j$. We claim that it has a fixed point. In fact, the group $K$ generated by $j$ and $H$ has the property that $S / K=R / J$ is a sphere with exactly 6 branch values of order two.

The elements of order two in $K$ are the liftings of $J$ and maybe one element of $H$. Since no element of $H$ has fixed points, we have that the only elements of order two in $K$ that may have fixed points are the liftings of $J$.

The covering induced by $K$ is of degree $2(g-1)$. The Riemann-Hurwitz formula asserts that on $S$ there are exactly $6(g-1)$ fixed points of order two.

Since each lifting of $J$ must have at most 6 fixed points on $S$, and there are exactly $g-1$ liftings, we have that every lifting has exactly 6 fixed points.

Now we can use the same argument to show that $h j$ has order two. In consequence, the group generated by $h$ and $j$ is a dihedral group of order $2(g-1)$.

\section{References}

[1] H. M. Farkas. Unramified coverings of hyperelliptic Riemann surfaces. Complex Analysis I, Lecture Notes in Math., vol. 1275, SpringerVerlag, New York, pp. 113-130, (1987).

[2] H. Farkas and I. Kra. Riemann surfaces. Springer-Verlag Graduate Texts in Mathematics, 71, Berlin, Heidelberg, New York, (1991).

[3] F. Harary. Graph theory. Addison-Wesley Series in Mathematics (1969).

[4] R. A. Hidalgo. On Schottky groups with automorphisms. Ann. Acad. Scie. Fenn. Ser. AI Mathematica 19, pp. 247-258, (1994). 
[5] R. A. Hidalgo. Schottky uniformizations of closed Riemann surfaces with abelian groups of conformal automorphisms. Glasgow Math. J. 36, pp. 17-32, (1994).

[6] R. A. Hidalgo. Closed Riemann surfaces with dihedral groups of conformal automorphisms. Revista Proyecciones 15, 47-90, (1996).

[7] S. P. Kerckhoff. The Nielsen realization problem. Ann. Math. 117, 235-265, (1983).

[8] B. Maskit. Kleinian groups. Grundlehren der Mathematischen Wissenschaften, Vol. 287, Springer - Verlag, Berlin, Heildelberg, New York, (1988).

[9] B. Maskit. On the classification of Kleinian groups I and II. Acta Math. 135 (1975) and 138 (1977).

[10] D. McCullough, A. Miller and B. Zimmermann. Group actions on handlebodies. Proc. London Math. Soc. 59, 373-416, (1989).

[11] J. F. X. Ries. Subvarieties of moduli space determined by finite group actions acting on surfaces. Transactions A.M.S. 335, 385-406, (1993).

[12] S. A. Wolpert. Geodesic length functions and the Nielsen problem. J. Diff. Geom. 25, 275-296, (1987).

[13] B. Zimmermann. Über Homöomorphismen n-dimensionaler Henkelkörper und endliche Erweiterungen von Schottky-Gruppen. Comment. Math. Helv. 56, 474-486, (1981).

[14] R. Rodríguez and V. González. On principally polarized abelian varieties induced by prysms and pyramids. To appear in Complex Geometry Seminar. Vol. IV (1995).

Received by : January 10, 1999.

\section{Rubén A. Hidalgo}

Departamento de Matemáticas

Universidad Técnica Federico Santa María

Casilla 110-V

Valparaíso

Chile 